\title{
Distributed beam forming with phase-only control for green cognitive radio networks
}

\author{
Xiaohua Lian", Homayoun Nikookar and Leo P Ligthart
}

\begin{abstract}
Cognitive radio (CR) is an intelligent radio system and is able to share the spectrum with licensed users (LU). By adopting adaptive beam forming techniques, CR can reuse the spectrum with LU via directing main beams towards CR users while displaying nulls towards LU. In this article, we present a new distributed beam forming (DB) technique, and study the performance of its application to decentralized CR networks. The presented DB method only controls the phase of the transmitted signal of each CR node in the CR network, and therefore, it is called phase-only DB (PODB) method. It can be implemented at each node independently with only prior knowledge of its own location, and directions of distant CR (DCR) users and LU. The average beam pattern and the average gains for an arbitrary number of CR nodes of PODB method are discussed, showing that CR nodes can constructively transmit signals to DCR users with less interferences to LU via employing the PODB method to form and direct main beams towards directions of DCR users while nulls towards directions of LU. PODB proves to be a green DB method by prolonging the lifetime of the CR network due to effective battery power consumptions at $C R$ nodes. The cumulative distributed function of the beam pattern for a large number of $C R$ nodes is derived and analysed to demonstrate that the PODB method increases the possibility that the transmitted power of the whole CR network at the directions of LU is lower than a certain threshold, which guarantees that CR network causes less disturbing effect to LU.
\end{abstract}

Keywords: distributed beam forming, collaborative beam forming, cognitive radio networks, phase-only distributed beam forming, null-steering distributed beam forming, green cognitive radio networks

\section{Introduction}

Cognitive radio $(\mathrm{CR})$ is a new concept radio presented in $[1,2]$, which is a promising approach to solve the intensive usage of the precious natural source-spectrum [3]. As described in [3], CR is capable of sensing the communication environment and adapting to it by adjusting its parameters. By applying a beam forming technique, CR can direct its main beams towards CR users while putting nulls to licensed users (LU) in uplink in order to share the same spectrum with LU without disturbing them $[4,5]$.

Distributed beam forming (DB), which is also referred to as collaborative beam forming, is originally employed as an energy-efficient scheme to solve the long distance transmission by wireless sensors networks (WSN), in

\footnotetext{
* Correspondence: x.lian@tudelft.nl

Microwave Technology and System for Radar (MTS-Radar), Department of Electrical Engineering, Mathematics and Computer Science, Delft University of Technology, Mekelweg 4, 2628CD Delft, The Netherlands
}

order to reduce the amount of required energy and consequently to extend the utilization time of the sensors. The basic idea of DB is that a set of nodes in wireless networks act as a virtual antenna array and then form a beam towards a certain direction to collaboratively transmit a signal. DB is proposed in [6] and it shows that by employing $K$ collaborative nodes, the collaborative beam forming can result in up to $K$-fold gain in the received power at a distance access point. A cross-layer approach to DB for wireless adhoc networks is discussed in [7] with more complicated models and two time phases of communication steps. The improved beam pattern and connectivity properties are shown in [8], and a reasonable beam forming performance affected by a node synchronization error is discussed in [9]. DB has also been introduced in relay communication systems [10-14]. In [10-13], several DB techniques for relay networks with flat fading channels have been developed, while in [14] frequency selective fading has been 
considered. DB requires accurate synchronization; in other words, the nodes must start transmitting at the same time, synchronize their carrier frequencies, and control their carrier phases so that their signals can be combined constructively at the destination. Several synchronization techniques for $\mathrm{CB}$ can be found in $[15,16]$, and a review is given in [17].

As discussed in $[4,5]$, the $\mathrm{CR}$ base station (BS) needs to be equipped with array antenna (AA) so that it can form and direct main beams to its own users while steering nulls towards the directions of LU via adaptive beam forming techniques. In this study, instead of requesting $\mathrm{CR}$ BS to be deployed together with antenna arrays, we consider decentralized CR networks, which is formed by distributed CR users in a certain area. Those $\mathrm{CR}$ users are therefore regarded as CR nodes. Meanwhile DB is suggested to be employed by the CR network to form beams towards distant CR (DCR) users so that the CR network is able to forward the signals to DCR users cooperatively. Thus, the whole network decreases the energy consumption effectively, and also increases the communication range.

However, the CR network distinguishes itself from WSN in that it is designed to achieve two goals under one restricted condition, i.e., the received power at DCR users should be maximized, while that at LU be minimized. The restriction limits that CR nodes are only aware of its own location, as well as the directions of DCR users and LU. The constraint supports DB for a CR network functional even without cluster nodes. If cluster nodes are selected, each CR node is able to have the information of locations of all CR nodes during the process of information exchanging and sharing. Then, the DB problem can be described as designing adaptive beam formers with an irregular AA structure. In this article, we consider no CR cluster nodes, which is the basic and simplest network application. For DB, the aim of a CR network can directly be translated into generating main beams towards DCR users while displaying nulls towards LU. In addition, the weights calculation can be performed by each node, and for each node it should be independent from the prior knowledge of other nodes due to the restriction introduced above. This article also suggests generating a certain angular range of nulls instead of point nulls towards LU. The reason is due to the study of the probability density function (pdf) of the angle of arrival (AOA) of the scattered wave at the mobile station in wireless communications [18-20], which will be explained later in this article.

Recently, DB for a CR network has also been discussed in [21-23]. In [21], the effect of noise in phase synchronization on the resultant beam pattern has been analysed. A DB scheme based on zero-forcing beam forming has been presented in [22], where the idea that a CR network can be regarded as a relay network to forward the signal to the destination CR node is adopted. It is worth noticing that in [23], the authors have also addressed the above problem of directing main beam to a required direction while putting nulls to unwanted directions. This fits well the idea of introducing DB to CR network. A novel null-steering DB method has been presented in [23] in a way that each node can calculate its own weight by itself without prior knowledge of others.

Despite that the DB weights derived in [23] achieve our goal of CR network successfully, further improvements of DB in application to green CR networks are appreciated. In this article, we present a phase-only DB (PODB) method by only controlling the phase of the transmitted signal of each node. With the proposed PODB method, the lifetime of the whole CR network is prolonged, which will be proved in this article.

The PODB method is able to direct main beams towards DCR users while nulls are formed towards directions of LU. It can be implemented independently by each node based on very limited knowledge, including directions of DCR users and LU, and its own location. The weight of the PODB method can be regarded as nothing more than a phase perturbation of each node, which has the same amplitude as that of others. By adding certain virtual directions, the proposed PODB method can also generate broadened nulls in a defined range around directions of LU. The average beam pattern is also derived to show what the beam pattern with an arbitrary number of collaborative nodes will converge to. The average gains at both directions of LU and DCR users are calculated approximately. What is more, the cumulative distributed function (CDF) of the beam pattern is derived; it demonstrates the possibility that the transmitted power of the whole CR network at every direction can be lower than a certain threshold. The lifetime of a wireless network with both null-steering DB which has been presented in [23] and the PODB method is discussed and compared, in order to show the "green" aspect of the latter method.

The rest of the article is organized as follows. First in Section 2, the model of the CR network will be presented, as well as several necessary assumptions. Then the PODB method is proposed with and without broadened nulls. The average beam pattern of the PODB method, and its gains at certain directions are discussed in Section 3, as well as the derived CDF of the beam pattern generated by the PODB method. Several simulation results are shown in Section 4. The greenness of the PODB method is illustrated in Section 5 by comparing the lifetime of the wireless networks adopting nullsteering DB and the PODB method. Finally, Section 6 concludes the article. 
Notations: $\|\mathbf{a}\|_{\infty},\|\mathbf{a}\|_{2}$ denote the infinitive norm and Euclidean norm of vector a. $(\cdot)^{T},(\cdot)^{*}$ and $(\cdot)^{H}$ denote the transpose, the conjugate, and the Hermitian transpose, respectively. (A) $m, n$ represents the element of the $m$ th row and $n$th column of matrix $\mathbf{A}$, and $(\mathbf{a})_{k}$ represents the $k$ th element of vector a. $E[\cdot]$ stands for the statistical expectation, and $\stackrel{P 1}{\rightarrow}$ denotes converge with probability one. $J_{n}(\cdot)$ is the $n$ th-order Bessel function of the first kind, and $I_{0}(\cdot)$ is the zeroth-order-modified Bessel function. $\operatorname{Re}[\cdot]$ and $\operatorname{Im}[\cdot]$ represent the real and imaginary parts of a variable, respectively.

\section{Problem formulations and the proposed PODB method}

\subsection{Geometrical structure of $\mathrm{CR}$ networks}

The geometrical structure of the CR network and also distant receiver terminals including LU and DCR users are illustrated in Figure 1. As shown in Figure 1, $K$ CR nodes are uniformly distributed on a disc centred at $O$ with radius $R$. Let us denote their polar coordinates of the $k$ th CR node as $\left(r_{k}, \Psi_{k}\right) . L$ DCR users are considered as access points, which locate in the same plane at $\left(A_{i}^{\mathrm{DCR}}, \phi_{i}^{\mathrm{DCR}}\right), i=1,2, \ldots, L$. Meanwhile, $M$ LU are also coexisting with DCR users. Their locations are $\left(A_{\dot{v}} \varphi_{i}\right), i$ $=1,2, \ldots, M$. The CR nodes in the CR network are requested to form a virtual antenna array and collaboratively transmit a common message $S(t)$.

\subsection{Necessary assumptions}

Without generality, we also adopt the following assumptions:
(1) The number of CR nodes are larger than that of DCR users together with LU, i.e. $K>L+M$. This is basically required to solve the later matrix equations. In addition, many adaptive beam forming techniques also request that the number of AAs is larger than that of constrained directions where main beams and nulls are dedicatedly directed towards.

(2) All DCR users and LU are located in the far-field of CR network, such that

$$
\begin{aligned}
& A_{i}^{D C R} \gg R, i=1,2, \ldots, L \\
& A_{i} \gg R, i=1,2, \ldots, M
\end{aligned}
$$

(3) The bandwidth of $S(t)$ is narrow, so that it guarantees that $S(t)$ is almost constant during $2 R / c$ second where $c$ is the speed of light. It has been discussed in [24] that OFDM scheme is a proper and recommended candidate for $C R$ due to its flexible adaptation of the spectrum. Since OFDM signal can be regarded as a combination of narrow-band modulated signals, the proposed method in this article can also be implemented in a wide band CR OFDM system.

The spatial effect of signal scattering and reflection at LU are taken into account. As a result, the angular spread at LU is suggested to be eliminated by displaying spread nulls around each direction of LU rather than point nulls. However, other channel effects, such as multipath fading and shadowing, are ignored in this article.

For simplicity, the proposed PODB is explained and presented when $L=1$. In case $L>1$, we show in [25] how to generate more than one main beam towards

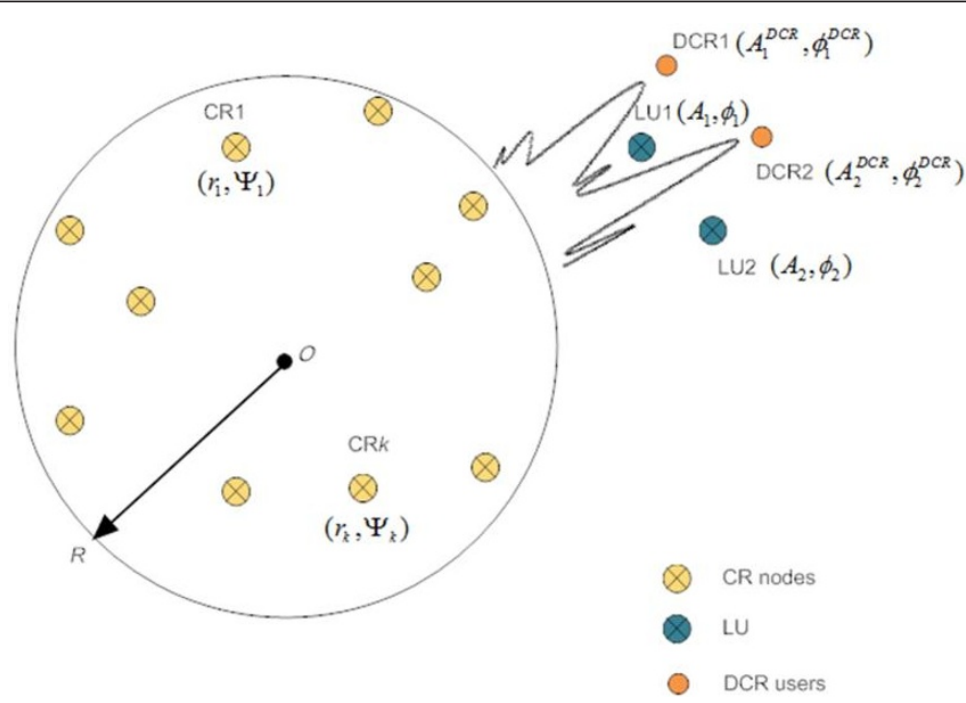

Figure 1 CR network with DCR users and LU 
DCR users. We simplify $\left(A_{1}^{\mathrm{DCR}}, \phi_{1}^{\mathrm{DCR}}\right)$ by $\left(A_{0}, \varphi_{0}\right)$ when there is only one DCR user.

\subsection{System model of CR network}

Let $x_{k}(t)$ denote the transmitted signal from $k$ th node,

$$
x_{k}(t)=S(t) e^{j 2 \pi f t} w_{k}
$$

where $w_{k}$ is the weight adopted by the $k$ th node. The received signal at an arbitrary point $(A, \varphi)$ in the farfield due to the $k$ th node transmission is [23],

$$
r_{k}(t)=\beta_{k} x_{k}\left(t-\frac{d_{k}}{c}\right)=\beta_{k} S\left(t-\frac{d_{k}}{c}\right) e^{j 2 \pi f t} e^{-j \frac{2 \pi}{\lambda} d_{k}} w_{k}
$$

where $d_{k}$ is the distance between the $k$ th node and the access point $(A, \varphi)$, and $\beta_{k}=\left(d_{k}\right) \frac{-\gamma}{2}$ is the signal path loss with $\gamma$ donating the path loss exponent. In [23], $\beta_{k}$ and $d_{k}$ are approximated by

$$
\begin{aligned}
& d_{k}=\sqrt{A^{2}+r_{k}^{2}-2 A r_{k} \cos \left(\phi-\Psi_{k}\right)} \approx A-r_{k} \cos \left(\phi-\Psi_{k}\right) \\
& \beta_{k}=\left(d_{k}\right) \frac{-\gamma}{2} \approx\left(A-r_{k} \cos \left(\phi-\Psi_{k}\right)\right) \frac{-\gamma}{2} \approx \beta\left(1+\frac{\gamma r_{k} \cos \left(\phi-\Psi_{k}\right)}{2 A}\right)
\end{aligned}
$$

where $\beta=A \frac{-\gamma}{2}$. The two approximations above are due to assumption 2. It also ensures $\frac{\gamma r_{k} \cos \left(\phi-\Psi_{k}\right)}{2 A} \ll 1$, and then $\beta_{k} \approx \beta$.

Substituting Equations (3) and (4) into Equation (2) it follows that [23],

$$
r_{k}(t) \approx \beta e^{-j \frac{2 \pi}{\lambda} A} S\left(t-\frac{A}{c}\right) e^{j 2 \pi f t} e^{j \frac{2 \pi}{\lambda} r_{k} \cos \left(\phi-\Psi_{k}\right)} w_{k}
$$

If we adopt the initial phase of each node as $\varphi_{k}=-\frac{2 \pi}{\lambda} r_{k} \cos \left(\phi_{0}-\Psi_{k}\right)[6]$, the received signal at $(A$, $\varphi)$ is

$$
r_{k}(t) \approx \beta e^{-j \frac{2 \pi}{\lambda} A} S\left(t-\frac{A}{c}\right) e^{j 2 \pi f e^{j}} e^{\frac{2 \pi}{\lambda} r_{k}\left[\cos \left(\phi-\Psi_{k}\right)-\cos \left(\phi_{0}-\Psi_{k}\right)\right]} w_{k}
$$

We define

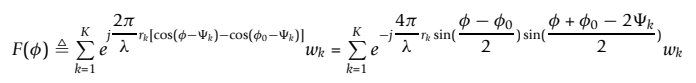

The power received at $(A, \varphi)$ is denoted by $p(\varphi)$. If $S(t)$ has normalized power, $p(\varphi)$ is shown as

$$
p(\phi)=\left|\sum_{k=1}^{K} r_{k}(t)\right|^{2}=\beta^{2}|F(\phi)|^{2}
$$

The goal of our proposed PODB method is to find $\mathbf{w}$, where $\mathbf{w}=\left(w_{1}, w_{2}, \ldots, w_{K}\right)^{T}$, which satisfies

$$
\left\{\begin{array}{c}
\max _{\mathbf{w}}\left|F\left(\phi_{0}\right)\right| \\
\left|F\left(\phi_{m}\right)\right|=0, m=1,2, \ldots, M \\
\text { subject to }\left|w_{k}\right|=1, k=1,2, \ldots, K
\end{array}\right.
$$

Based on Equation (7), it is easy to verify that $\frac{d F(\phi)}{d \phi}=0$. Therefore, $\max _{\mathbf{w}}\left|F\left(\phi_{0}\right)\right|$ is always satisfying.

The constraint in (9) shows that each $w_{k}$ is no more than a phase adjustment. In other words, we only control the phase modification of each node.

A different optimization problem is discussed in [23], which has been proposed based on null-steering beamformers. It has the constraint of the total power of $\mathbf{w}, \mathrm{i}$. e. $\mathbf{w}^{H} \mathbf{w} \leq P_{T}$, instead of $\left|w_{i}\right|=1, i=1,2, \ldots, K$.

\subsection{The proposed PODB method}

We propose the solution of Equation (9) in this section. First we assume $W=0$, where $W$ is the width of the spread nulls towards each LU, which requires only a point null at each LU direction, and then we give further results of how to generate spread nulls by modifying the angles.

Equation (9) also appeared in the application of adaptive array techniques to solve the problems of array pattern nulling, which was studied in [26]. The proposed solution in [26] linearized the nonlinear problem by considering small position perturbation of each antenna element, and then derived analytic results. It was also proved in [26] that good nulling performance could be achieved, even though the solutions were based on approximations. Unfortunately, the results shown in [26] could be only adopted by uniformly linear arrays (ULA).

In the case of CR network, the structure and the motivation of the beam forming problem are very different from that in [26]. As mentioned before, our goal is to find a set of weights $\left(w_{1}, w_{2}, \ldots, w_{K}\right)$, which can be implemented by each node under the constraint that only the information of $\left(r_{k}, \Psi_{k}\right)$, the directions of DCR $\left(\varphi_{0}\right)$ and $\mathrm{LU}\left(\varphi_{i}, i=1,2, \ldots, M\right)$ are available.

We assume that $w_{k}$ has the form of

$$
w_{k}=e^{j \mu_{k}}
$$

Then, we perform a Taylor expansion in Equation (7) and retain the first two terms. We obtain 


$$
\begin{aligned}
& F(\phi)=\sum_{k=1}^{K} e^{-j \frac{4 \pi}{\lambda} r_{k} \sin \left(\frac{\phi+\phi_{0}-\Psi_{k}}{2}\right) \sin \left(\frac{\phi-\phi_{0}}{2}\right)+j \mu_{k}} \\
& \approx \sum_{k=1}^{K} e^{-j \frac{4 \pi}{\lambda} r_{k} \sin \left(\frac{\phi+\phi_{0}-\Psi_{k}}{2}\right) \sin \left(\frac{\phi-\phi_{0}}{2}\right)}+j \sum_{k=1}^{K} \mu_{k} e^{-j \frac{4 \pi}{\lambda} r_{k} \sin \left(\frac{\phi+\phi_{0}-\Psi_{k}}{2}\right) \sin \left(\frac{\phi-\phi_{0}}{2}\right)(11)} \\
& \approx F_{0}(\phi)+F_{C}(\phi)
\end{aligned}
$$

$F_{C}(\varphi)$ represents the second item of (11) and is a cancellation pattern which can be used to achieve $M$ nulls.

Let we define the following variables

$$
\begin{aligned}
& \boldsymbol{\mu} \triangleq\left(\mu_{1}, \mu_{2} \quad \cdots, \mu_{K}\right)^{T} \\
& \mathbf{u}_{\bullet} \triangleq\left(e^{-j \frac{4 \pi}{\lambda} r_{1} \sin \left(\frac{\phi_{\bullet}+\phi_{0}-\Psi_{1}}{2}\right) \sin \left(\frac{\phi_{\bullet}-\phi_{0}}{2}\right)} \cdots e^{-j \frac{4 \pi}{\lambda} r_{\kappa} \sin \left(\frac{\phi_{\bullet}+\phi_{0}-\Psi_{K}}{2}\right) \sin \left(\frac{\phi_{\bullet}-\phi_{0}}{2}\right)}\right)^{T}=\mathbf{x}_{\bullet}+j \mathbf{y}_{\bullet}
\end{aligned}
$$

where $\boldsymbol{\mu} \in \mathfrak{R}^{K \times 1}, \mathbf{x}_{\bullet}=\operatorname{Re}\left[\mathbf{u}_{\text {. }}\right.$, and $\mathbf{y}_{\mathbf{0}}=\operatorname{Im}\left[\mathbf{u}_{\text {. }}\right]$.

$$
\begin{aligned}
& \alpha_{\bullet, 0} \triangleq 4 \pi \frac{R}{\lambda} \sin \frac{\phi_{\bullet}-\phi_{0}}{2} \\
& \alpha_{m, n} \triangleq 4 \pi \frac{R}{\lambda} \sin \frac{\phi_{m}-\phi_{n}}{2} \\
& \alpha_{\bullet, m} \triangleq 4 \pi \frac{R}{\lambda} \sin \frac{\phi_{\bullet}-\phi_{m}}{2} \\
& \mathbf{e}=(1,1, \ldots, 1)^{T}
\end{aligned}
$$

where $\mathbf{e}$ is a $K \times 1$ vector with one as its entities.

Thus, Equation (11) can be simplified as

$$
F\left(\phi_{\bullet}\right)=\mathbf{e}^{H} \mathbf{u}_{\bullet}+j \boldsymbol{\mu}^{H} \mathbf{u}_{\bullet}
$$

According to Equation (9), when $W=0$, in order to steer nulls at direction of $\varphi_{m},=1,2, \ldots, M$, we require

$$
F\left(\phi_{m}\right)=0, m=1,2, \ldots, M
$$

Due to assumption 1, Equation (13) has a solution and can be solved as the following. Using the results in [6],

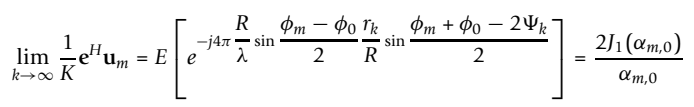

Equation (13) can be further written as

$$
\left\{\begin{array}{c}
\boldsymbol{\mu}^{H} \mathbf{x}_{m}=0 \\
K \frac{2 J_{1}\left(\alpha_{m, 0}\right)}{\alpha_{m, 0}}=\boldsymbol{\mu}^{H} \mathbf{y}_{m}, \quad m=1,2, \ldots, M
\end{array}\right.
$$

If we define

$$
\begin{aligned}
& X \triangleq\left(\mathbf{x}_{1}, \mathbf{x}_{2}, \ldots, \mathbf{x}_{M}\right) \\
& Y \triangleq\left(\mathbf{y}_{1}, \mathbf{y}_{2}, \ldots, \mathbf{y}_{M}\right)
\end{aligned}
$$

$$
\begin{gathered}
\Delta \triangleq(\mathrm{X} \mathrm{Y}) \\
\boldsymbol{\Gamma}=\Delta^{H} \Delta \\
\mathbf{c} \triangleq\left(c_{1}, c_{2}, \ldots, c_{M}\right) \\
\text { where } c_{m}=\frac{2 J_{1}\left(\alpha_{m, 0}\right)}{\alpha_{m, 0}} .
\end{gathered}
$$

The solution of Equation (15) is

$$
\boldsymbol{\mu}^{H}=K(\mathbf{0} \mathbf{c}) \boldsymbol{\Gamma}^{-1} \Delta^{H}
$$

where $(\boldsymbol{O})$ is a vector with length $M$ and $O$ as its entries.

To make the calculation of $\boldsymbol{\mu}$ be able to be implemented by each node in a distribution fashion, we need to calculate $\boldsymbol{\Gamma}$ differently, which has a good approximation of original counterparts, and the entries of it depends only on the information that each node is entitled to have $\left(r_{k}, \Psi_{k}\right), \varphi_{0}, \varphi_{m}, m=1,2, \ldots, M$.

Theorem 1: Give a matrix $\mathbf{U}$, which is defined as

$\mathbf{U} \triangleq\left(\mathbf{u}_{1}, \mathbf{u}_{2}, \ldots, \mathbf{u}_{M}\right)=\mathbf{X}+j \mathbf{Y}$, we have the following results:

$$
\begin{aligned}
& \lim _{k \rightarrow \infty} \frac{1}{K}\left(\mathbf{U}^{H} \mathbf{U}\right)_{m, n} \stackrel{P_{1}}{\rightarrow} E\left[\frac{\mathbf{u}_{m}^{H} \mathbf{u}_{n}}{K}\right]=E\left[\left(\mathbf{u}_{m}^{*}\right)_{k}\left(\mathbf{u}_{n}\right)_{k}\right]=\left\{\begin{array}{c}
1, m=n=1,2, \ldots, M \\
\frac{2 J_{1}\left(\alpha_{m, n}\right)}{\alpha_{m, n}}, m \neq n ; m, n=1,2, \ldots, M
\end{array}\right. \\
& \lim _{k \rightarrow \infty} \frac{1}{K}\left(\mathbf{U}^{H} \mathbf{U} *\right)_{m, n} \stackrel{P 1}{\rightarrow} E\left[\frac{\mathbf{u}_{\mathbf{u}_{n}}^{H} \mathbf{u}_{n}^{*}}{K}\right]=E\left[\left(\mathbf{u}_{m}^{*}\right)_{k}\left(\mathbf{u}_{n}^{*}\right)_{k}\right]=\left\{\begin{array}{c}
\frac{J_{1}\left(2 \alpha_{m, 0}\right)}{\alpha_{m, 0}}, m=n=1,2, \ldots, M \\
\frac{2 J_{1}\left(\tilde{\alpha}_{m, n}\right)}{\tilde{\alpha}_{m, n}}, m \neq n ; m, n=1,2, \ldots, M
\end{array}\right.
\end{aligned}
$$

where $\tilde{\alpha}_{m, n}=4 \pi \frac{R}{\lambda} \sqrt{1+\cos ^{2} \frac{\phi_{m}-\phi_{n}}{2}-\cos \left(\phi_{0}-\phi_{n}\right)-\cos \left(\phi_{0}-\phi_{m}\right)}$.

Proof: See Appendix 1

Theorem 2: The entries of $\Gamma$ have the following results:

$$
\lim _{k \rightarrow \infty} \frac{1}{K} \boldsymbol{\Gamma} \stackrel{P 1}{\rightarrow} E\left[\frac{\boldsymbol{\Gamma}}{K}\right]=\left(\begin{array}{cc}
\left(\boldsymbol{\Gamma}_{1}\right)_{M \times M} & \mathbf{0} \\
\mathbf{0} & \left(\boldsymbol{\Gamma}_{2}\right)_{M \times M}
\end{array}\right)
$$

$$
\begin{gathered}
\left(\boldsymbol{\Gamma}_{1}\right)_{m, n}=\left\{\begin{array}{c}
\frac{J_{1}\left(2 \alpha_{m, 0}\right)}{2 \alpha_{m, 0}}+\frac{1}{2}, m=n=1,2, \ldots, M \\
\frac{J_{1}\left(\tilde{\alpha}_{m, n}\right)}{\tilde{\alpha}_{m, n}}+\frac{J_{1}\left(\alpha_{m, n}\right)}{\alpha_{m, n}}, m \neq n ; m, n=1,2, \ldots, M
\end{array}\right. \\
\left(\boldsymbol{\Gamma}_{2}\right)_{m, n}=\left\{\begin{array}{c}
\frac{1}{2}-\frac{J_{1}\left(2 \alpha_{m, 0}\right)}{2 \alpha_{m, 0}}, m=n=1,2, \ldots, M \\
\frac{J_{1}\left(\alpha_{m, n}\right)}{\alpha_{m, n}}-\frac{J_{1}\left(\tilde{\alpha}_{m, n}\right)}{\tilde{\alpha}_{m, n}}, m \neq n ; m, n=1,2, \ldots, M
\end{array}\right.
\end{gathered}
$$

Proof: See Appendix 2

Based on Theorem 2, we can replace $\Gamma$ by $E[\boldsymbol{\Gamma}]$, and then calculate $(E[\boldsymbol{\Gamma}])^{-1}$ by 


$$
(E[\boldsymbol{\Gamma}])^{-1}=\frac{1}{K}\left(\begin{array}{cc}
\boldsymbol{\Gamma}_{1}^{-1} & \mathbf{0} \\
\mathbf{0} & \boldsymbol{\Gamma}_{2}^{-1}
\end{array}\right)
$$

If we define

$$
\mathbf{c} \boldsymbol{\Gamma}_{2}^{-1} \triangleq\left(q_{1}, q_{2}, \ldots, q_{M}\right)
$$

Then by combining (16) and (22), we have

$$
\boldsymbol{\mu}^{H}=\mathbf{c} \boldsymbol{\Gamma}_{2}^{-1} \mathbf{Y}^{H}
$$

The weight of the $k$ th CR node is represented by

$$
w_{k}=e^{j \mu_{k}}=e^{j(\mu)_{k}}=e^{\left(\sum_{m=1}^{M} j q_{m}\left(\mathbf{y}_{m}\right)_{k}\right)}
$$

So far we have derived the weight of the PODB method, which shows that the computation of $w_{k}$ for each node only depends on $\mathbf{c}, \boldsymbol{\Gamma}_{2}$, and $\left(\mathbf{y}_{m}\right)_{k}$. The first two can be regarded as constant vectors and a constant matrix, as shown in their definitions and Theorem 2 . While in order to calculate $\left(\mathbf{y}_{m}\right)_{k}$, each node only requires the prior knowledge of its own location $\left(r_{k}, \Psi_{k}\right)$, and directions of all $\mathrm{LU} \varphi_{m}, m=1,2, \ldots, M$. It is worth noticing that the information of the direction of DCR user $\varphi_{0}$ is also needed for deciding the initial phase of each node $\phi_{k}$ in Equation (6).

\subsection{PODB method with spread nulls}

The principle of the necessity of generating spread nulls has been explained in [25]. In wireless communication systems, the received signal and power spectra at the mobile station at wireless communication depend on the pdf of the AOA of the scattered wave. Clarke considered a uniform AOA pdf over $[-\pi, \pi)$ [18]. However, it has been argued and experimentally demonstrated that the scattering encountered in many environments results into a non-uniform pdf of AOA [19]. Reference [20] suggests the two-parameter Von Mises pdf as a flexible and generalized model for the pdf of AOA, which includes non-isotropic scattering cases, and also the isotropic one as a special case. The pdf is given as [20]

$$
p_{\Theta}(\theta)=\frac{\exp \left(l \cos \left(\theta-\theta_{p}\right)\right)}{2 \pi I_{0}(l)}, \quad \theta \in[-\pi, \pi)
$$

where $\theta_{p}$ accounts for the main direction of the AOA scatter components. Parameter $l \geq 0$ controls the width of the AOA of scatter components. Figure 2 shows the $p_{\Theta}(\theta)$ with different $l$ for $\theta_{p}=0$. Based on Von Mises model, point nulls which can be generated by CR networks are not sufficient enough for energy depression at directions of LU, because the power around the nulling direction may also leak into LU due to spatial scattering. As a result, instead of generating point nulls, CR network is required to display spread nulls around each direction of the LU.

Null broadening (NB) technique was originally developed as a robust array beam forming technique. It was also regarded as a beam pattern synthesis method. Mailloux [27] presented a NB method by simple modification of the covariance matrix of the received signal. While in [28], a similar NB algorithm was also presented by applying a transform to the same covariance matrix. Both methods were capable of providing notch at locations of interferences signals. In [29], two NB algorithms as covariance matrix taper methods are defined, and considered as effective robust adaptive beam forming techniques, imparting robustness into adaptive pattern by judicious choice of null placement and width. As for wireless communication, NB was employed in a cellular communication system, particularly an example was given in space division multiple access system for downlink beam forming [30].

The above proposed NB methods are all based on ULA. They all require the prior knowledge of the distance between every two AA elements. In our case, this requested information can be translated into the distance between every two CR nodes in CR networks. Consequently, this does not align with our constraint that each node is only aware of its own location.

However, one simple way of generating spread nulls is to add $N$ virtual LU sources $\varphi_{i}, i=1,2, \ldots, N$ around each direction of existing $\mathrm{LU}$ with the angle range of $W / 2$, e. g. $\left|\varphi_{i}-\varphi_{m}\right| \leq W / 2, m=1,2, \ldots, M$. The number of those virtual directions $N$ can be chosen with a compromising between computation load and the depth of spread nulls. If $N$ is large enough, all the angles around directions of the LU are continuously all regarded as nulling points, thus the spread nulls in the beam pattern are maintaining the same depth with that of the nulling point $\varphi_{m}$. However, with large $N$, the size of matrix $\boldsymbol{\Gamma}_{2}$ in Theorem 2 increases to $(M N) \times(M N)$, and consequently each node must have larger computation burden.

\section{Properties of the beam pattern generated by the PODB method}

In this section, we discuss the properties of the beam pattern that generated by the presented PODB method. The analysed properties include the average beam pattern for an arbitrary number of CR nodes $K$, the average gains at the directions of DCR user $\varphi_{0}$, and at those of all the LU $\varphi_{m}, m=1,2, \ldots, M$. Meanwhile the CDF of the beam pattern with larger $K$ is also derived.

\subsection{Average beam pattern}

The result of average beam pattern is given by Theorem 3. 


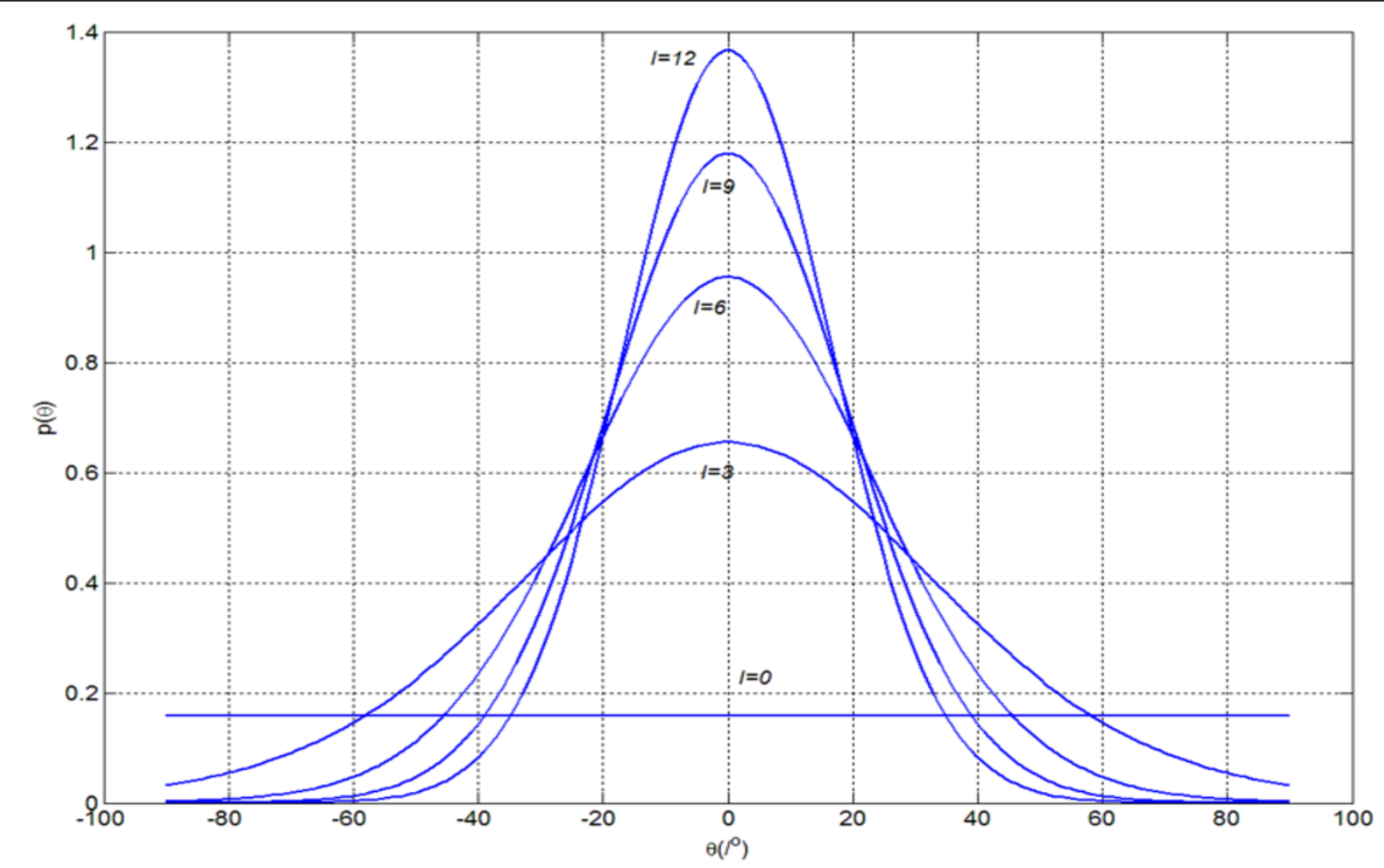

Figure 2 Von Mises pdf for the AOA of scatter components at mobile station

Theorem 3: Using the presented PODB method, the average beam pattern, which is defined as

$$
\tilde{P}\left(\phi_{\bullet}\right) \triangleq E\left[P\left(\phi_{\bullet}\right)\right]=E\left[\left|\frac{1}{K} F\left(\phi_{\bullet}\right)\right|^{2}\right]
$$

when $\varphi_{.} \neq \varphi_{0}$, can be approximated by

$$
\tilde{P}\left(\phi_{\bullet}\right) \approx \tilde{P}_{a}\left(\phi_{\bullet}\right)=\frac{1}{K}+\frac{K-1}{K}\left[\left(\frac{2 J_{1}\left(\alpha_{\boldsymbol{\bullet}, 0}\right)}{\alpha_{\boldsymbol{\bullet}, 0}}\right)^{2}+\frac{J_{1}\left(\alpha_{\boldsymbol{\bullet}, 0}\right)}{\alpha_{\boldsymbol{\bullet}, 0}} \sum_{m=1}^{M} q_{m} \zeta_{\bullet, m}\right]
$$

where

$\zeta_{\bullet}, m=\left\{\begin{array}{c}\frac{J_{1}\left(2 \alpha_{m, 0}\right)}{\alpha_{m, 0}}-1, \phi_{\bullet}=\phi_{m}, m=1,2, \ldots, M \\ \frac{2 J_{1}\left(\tilde{\alpha}_{\bullet, m}\right)}{\tilde{\alpha}_{\bullet, m}}-\frac{2 J_{1}\left(\alpha_{\bullet}, m\right)}{\alpha_{\bullet}, m}, \phi_{\bullet} \neq \phi_{m}, m=1,2, \ldots, M\end{array}\right.$

and the approximation error $\varepsilon=E\left[\left|\tilde{P}\left(\phi_{\bullet}\right)-\tilde{P}_{a}\left(\phi_{\bullet}\right)\right|\right]$ is bounded by $\varepsilon \leq \frac{K-1}{K} \mathbf{c \Gamma}_{2}^{-1} \mathbf{c}^{H}$.

Proof: See Appendix 3

\subsection{Average gain at direction of DCR}

The average gain at $\varphi_{0}$ is approximated by 1 , i.e.

$$
\tilde{P}\left(\phi_{0}\right) \approx 1
$$

Proof: See Appendix 4

\subsection{Average gains at directions of $\mathrm{LU}$}

The average gain at $\varphi_{m}, m=1,2, \ldots, M$ is

$$
\tilde{P}\left(\phi_{m}\right) \approx \frac{1}{K}, m=1,2, \ldots, M
$$

\section{Proof: See Appendix 5}

For CR network, it is very important to limit the transmission power at directions of LU. From Equation (27) and (28), we can see that towards LU directions, the average transmission power are $K-1$ times lower than that towards the direction of DCR user.

When there is no LU available, i.e. $M=0$. Equation (23) gives the result of $\mu_{k}=0$. Consequently,

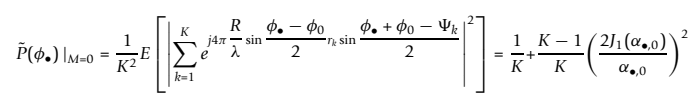

This is consistent with the average beam pattern of the DB method presented in [6], which has no specific nulling points.

3.4. CDF of the beam pattern generated by PODB method The CDF of the beam pattern generated by PODB method with large number of $K$ is given by Theorem 4 . 


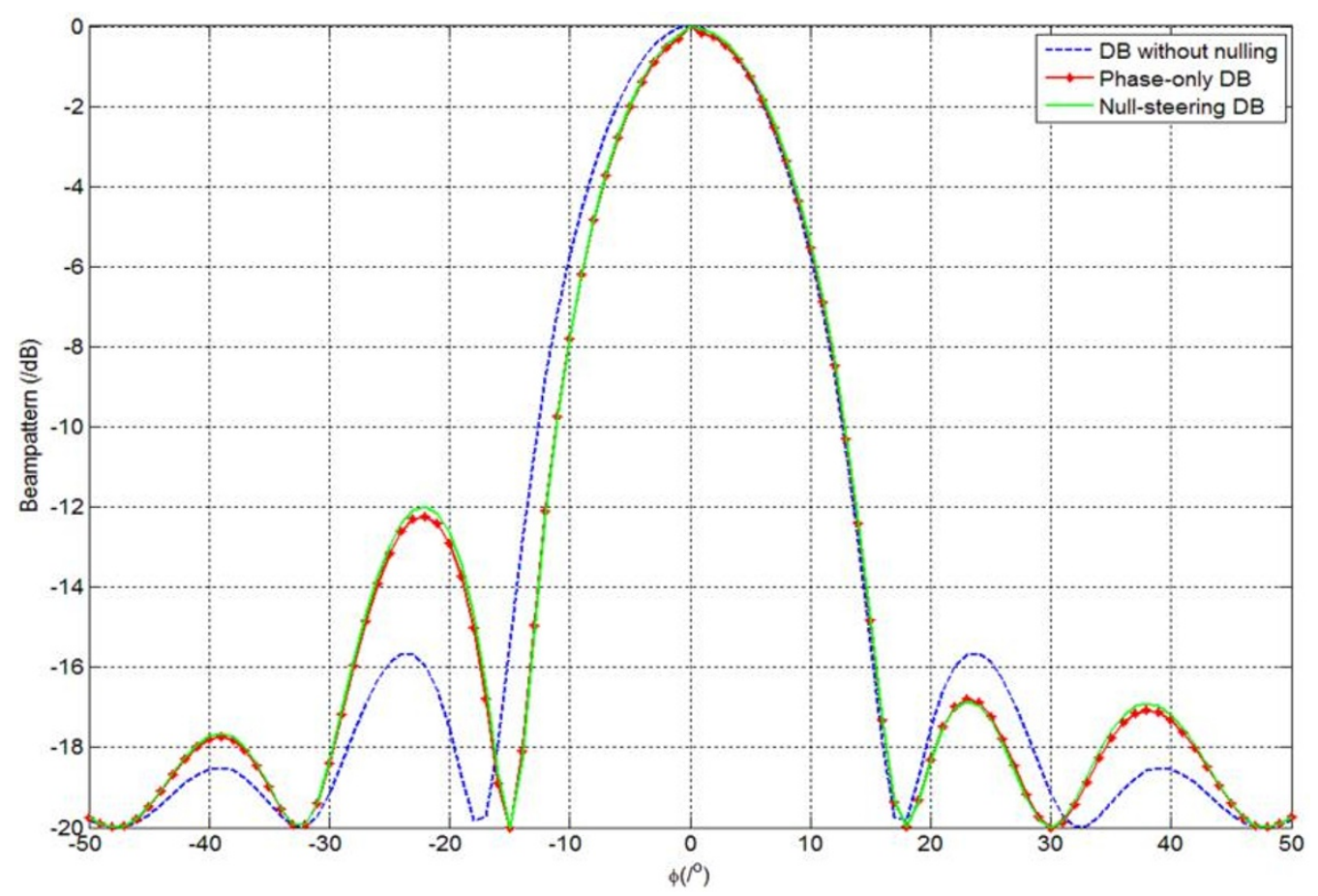

Figure 3 Average Beampatterns of three DB methods.

Theorem 4: When $K$ is large enough, the CDF of the beam pattern at different $\varphi$. can be approximated by

$$
\operatorname{Prob}\left\{P\left(\phi_{\bullet}\right) \mid P\left(\phi_{\bullet}\right) \leq P_{0}\right\} \approx F\left(|\rho|+\sqrt{P_{0}}\right)-\frac{\operatorname{sgn}\left(|\rho|-\sqrt{P_{0}}\right)+1}{2} F\left(|\rho|-\sqrt{P_{0}}\right)
$$

where $\quad F\left(\gamma_{0}\right)=\operatorname{Prob}\left(\gamma \mid \gamma \leq \gamma_{0}\right)=1-\exp \left[-\gamma_{0}^{2} K /\left(1+\mathbf{c} \boldsymbol{\Gamma}_{2}^{-1} \mathbf{c}^{H}-\rho^{2}\right)\right]$ for $\gamma \geq 0$ is the CDF of the Rayleigh distribution, $\operatorname{sgn}(x)$ $=|x| / x$ and $\rho=\frac{2 J_{1}\left(\alpha_{\bullet, 0}\right)}{\alpha_{\bullet}, 0}+\sum_{m=1}^{M} \frac{q_{m}}{2} \varsigma_{\bullet, m}$.

Proof: See Appendix 6.

We can write when $\varphi_{\bullet}=\varphi_{m}, m=1,2, \ldots, M$

$$
\operatorname{Prob}\left\{P\left(\phi_{m}\right) \mid P\left(\phi_{m}\right) \leq P_{0}\right\} \approx F\left(\sqrt{P_{0}}\right)=1-\exp \left[-P_{0} K /\left(1+\mathbf{c}_{2}^{-1} \mathbf{c}^{H}\right)\right]
$$

Proof: See Appendix 7.

From the above equation, we can conclude that when $\varphi_{.}=\varphi_{m}, m=1,2, \ldots, M$, the probability of the total transmitted power of CR network lower than $P_{0}$ can be expressed by a constant, which is decided by $P_{0}, K$ and $\mathbf{c} \boldsymbol{\Gamma}_{2}^{-1} \mathbf{c}^{H}$. Based on Equation (21) and the definition of $\mathbf{c}$, $\mathbf{c} \boldsymbol{\Gamma}_{2}^{-1} \mathbf{c}^{H}$ can be only determined by $\varphi_{0}, \varphi_{m}, m=1,2, \ldots, M$ and $R / \lambda$. Usually $P_{0}$ is defined by LU due to its spectrum interference tolerance threshold. Consequently, if we want to increase the probability of the case that CR network transmitting less power than $P_{0}$ towards directions of the LU, we need to increase $K$. In other words, more $C R$ nodes need to be added to the same CR network (maintaining the same network structure $R / \lambda$ ) to collaboratively perform PODB.

\section{Simulations and results}

The average beam pattern of DB methods are shown in Figure 3. They are DB method without considering nulling directions, which has been presented in [6], the proposed PODB method, and null-steering DB method in [23]. In the simulation, we choose $R / \lambda=2, K=100$ and $\varphi_{0}=0^{\circ}$. Meanwhile, two LU are considered, which locate at the directions of $\varphi_{1}=30^{\circ}, \varphi_{2}=-15^{\circ}$. Figure 3 shows that PODB and null-steering DB methods successfully depress transmission power towards the directions of LU. Their performances are similar to each other. The DB method without nulling has a symmetrical average beam pattern without particular nulling points. As mentioned in Equations (27) and (28), the average gain at $\varphi_{0}=0^{\circ}$ approximates to 1 , while those at $\varphi_{1}=30^{\circ}, \varphi_{2}=-15^{\circ}$ are close to $10 \log (1 / K)=-20 \mathrm{~dB}$.

Figure 4 shows the average beam pattern with only one LU, which has the direction of $\varphi=30^{\circ}$. The other simulation conditions remain the same with Figure 3. In Figure 4, we also consider the average beam pattern of PODB method with broadened nulls, which assumes two virtual LU directions around $\varphi=30^{\circ}$, which are located at $\varphi_{1}=20^{\circ}, \varphi_{2}=40^{\circ}$. It can be seen from Figure 4 that PODB method with NB generates spread nulls 


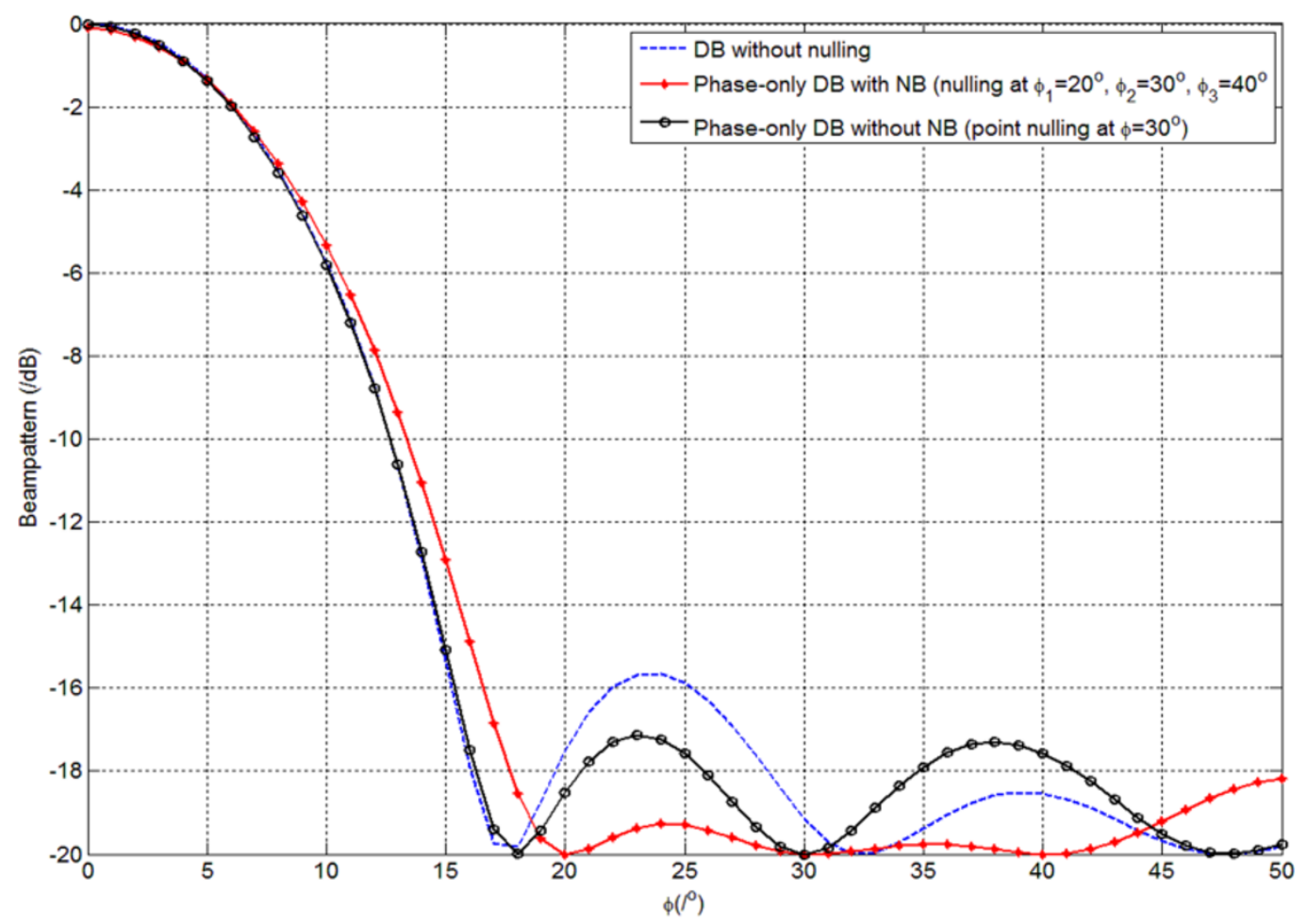

Figure 4 Average Beampatterns of PODB methods.

around $\varphi=30^{\circ}$ with the width of $W=20^{\circ}$ and depth about $-20 \mathrm{~dB}$.

Figure 5 shows the CDF of the beam pattern at different angle $\varphi$, which has been discussed in Theorem 4. As required in Theorem 4, the number of nodes in CR network needs to be large, so that the sum of random variables can be regarded as Gaussian distribution random variables. Therefore, we consider two cases with $K=100$, and $K=500$. Meanwhile, only one LU is considered, i.e. $\varphi=5^{\circ}$. The DCR user is at the direction of $\varphi_{0}=0^{\circ} . P_{0}$ in Theorem 4 is chosen as $P_{0}=$ 0.01. It can be seen from Figure 5 that, in the angle range of $5^{\circ} \leq \varphi \leq 7^{\circ}$, considering the probability that the transmitted power of CR network lower than $P_{0}$, PODB method has much higher chance than the DB method without nulling. Meanwhile, as discussed in Section 3.4, $\operatorname{Prob}\left\{P\left(\varphi_{m}\right) \mid P\left(\varphi_{m}\right) \leq P_{0}\right\}$ is a constant which depends on $\varphi_{1}, \varphi_{0}$ and $K$. When more nodes participate in CR network to perform DB ( $K$ is increasing), $\operatorname{Prob}\left\{P\left(\varphi_{m}\right) \mid P\left(\varphi_{m}\right) \leq P_{0}\right\}$ is also increasing, which is also demonstrated in Figure 5.

\section{Greenness of the PODB method}

The battery consumptions of the network adopting the proposed PODB method and null-steering DB method presented in [23] are illustrated in Figures 6 and 7. Figure 6 shows that to perform PODB, each node of CR network consumes the same amount of battery power at the same time. The power consumption of the whole network is "balanced", because of the constraint in Equation (9), i.e. $\left|w_{k}\right|=1$. While null-steering DB method utilizes the battery power of each node "unevenly", as shown in Figure 7. When the battery of one node is dying, the others nodes are still power supplied.

For null-steering method, we introduce a new concept of "DB cycle". It describes the process from the moment that all nodes in the network start participating DB till the moment that one node in the network first runs out of its battery. Then, the rest of the nodes of the network will start computing the new weights of DB method with one less number of the nodes, and thus another round of DB cycle begins.

The number of CR nodes $K$ in a DB cycle is requested to satisfy $k \geq K_{0}, 1 \leq K_{0} \leq K$, where $K_{0}$ is the least number of $C R$ nodes required by $C R$ network to perform $\mathrm{DB}$, due to the restriction of the transmission power towards directions of LU

$$
\tilde{P}\left(\phi_{m}\right)=\frac{1}{k} \leq \frac{1}{K_{0}}, m=1,2, \ldots, M
$$

We denote the normalized the weights of the $i$ th DB cycle of null-steering DB method as $\mathbf{w}_{\mathrm{NS}}^{(i)}$ and it satisfies 


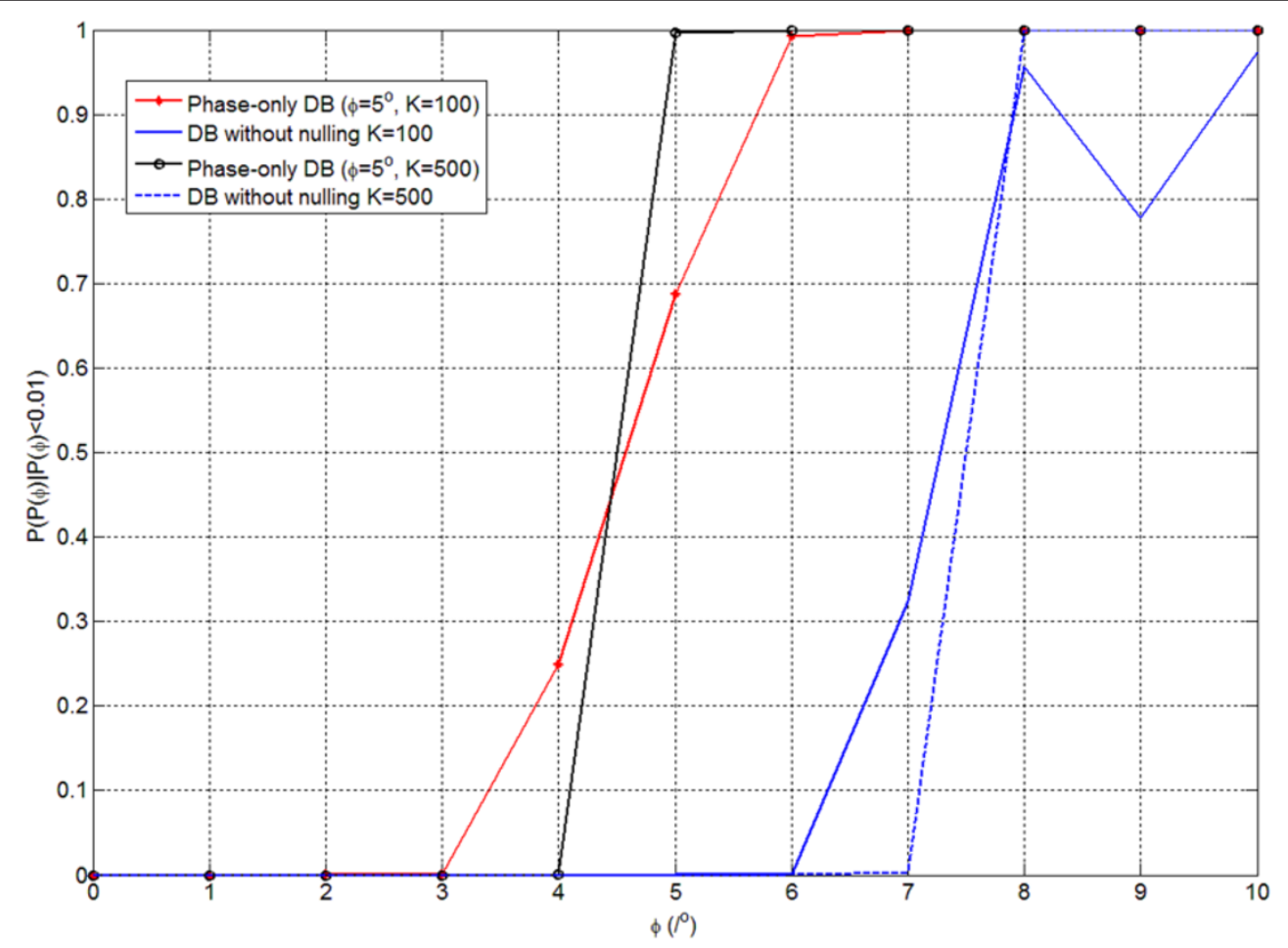

Figure 5 Probability of with different $K$.

that $\left(\mathbf{w}_{\mathrm{NS}}^{(i)}\right)^{H} \mathbf{w}_{\mathrm{NS}}^{(i)}=1$. Assume $t_{\mathrm{NS}}^{(i)}$ is the length of the time period of the $i$ th DB cycle of the null-steering DB method

$$
t_{\mathrm{NS}}^{(i)}=\frac{p^{(i)}}{\max _{k}\left\{\left|\left(\mathbf{w}_{\mathrm{NS}}^{(i)}\right)_{k}\right|^{2}\right\}}=\frac{p^{(i)}}{\left(\left\|\mathbf{w}_{\mathrm{NS}}^{(i)}\right\|_{\infty}\right)^{2}}
$$

where $p^{(i)}$ is the battery power consumption of the CR node, which finishes its battery first in the $i$ th DB cycle. Then, the total lifetime of CR network by adopting nullsteering method can be written as

$$
t_{\mathrm{NS}}=\sum_{i=1}^{K-K_{0}+1} \frac{p^{(i)}}{\left(\left\|\mathbf{w}_{N S}^{(i)}\right\|_{\infty}\right)^{2}}
$$

The total energy consumption is

$$
E_{N S}=\sum_{i=1}^{K-K_{0}+1} \frac{p^{(i)}}{\left(\left\|\mathbf{w}_{\mathrm{NS}}^{(i)}\right\|_{\infty}\right)^{2}}\left(\left\|\mathbf{w}_{\mathrm{NS}}^{(i)}\right\|_{2}\right)^{2}=\sum_{i=1}^{K-K_{0}+1} \frac{p^{(i)}}{\left(\left\|\mathbf{w}_{\mathrm{NS}}^{(i)}\right\|_{\infty}\right)^{2}}
$$

The total amount of energy of the whole network is fixed, due to the fact that all nodes will consume all of their batteries power in the end. Let us denote the total energy of the CR network as $E$.

$$
E=\sum_{i=1}^{K} \frac{p^{(i)}}{\left(\left\|\mathbf{w}_{\mathrm{NS}}^{(i)}\right\|_{\infty}\right)^{2}}\left(\left\|\mathbf{w}_{\mathrm{NS}}^{(i)}\right\|_{2}\right)^{2}=\sum_{i=1}^{K} \frac{p^{(i)}}{\left(\left\|\mathbf{w}_{\mathrm{NS}}^{(i)}\right\|_{\infty}\right)^{2}}
$$

It is easy to verify that

$$
E_{\mathrm{NS}} \leq E
$$

Only when $K_{0}=1$,

$$
E_{\mathrm{NS}}=E
$$

However, the concept of DB cycle is not necessary for PODB, because all the nodes will be finishing their batteries at the same time. We define the normalized weights of the PODB method as $\mathbf{w}_{\mathrm{po}}$, and it satisfies $\mathbf{w}_{\mathrm{PO}}^{H} \mathbf{w}_{\mathrm{PO}}=1$. Then, the lasting time of the network with the PODB method using the same amount of energy $E$ is

$$
t_{\mathrm{PO}}=\frac{E}{K} \frac{1}{\max _{k}\left\{\left|\left(\mathbf{w}_{\mathrm{PO}}\right)_{k}\right|^{2}\right\}}=\frac{E}{K\left(\left\|\mathbf{w}_{\mathrm{PO}}\right\|_{\infty}\right)^{2}}=\frac{E}{K} K=E
$$

Using the results of Equations (34), (35), (37) and (39), we can write that

$$
t_{\mathrm{NS}} \leq t_{\mathrm{PO}}
$$




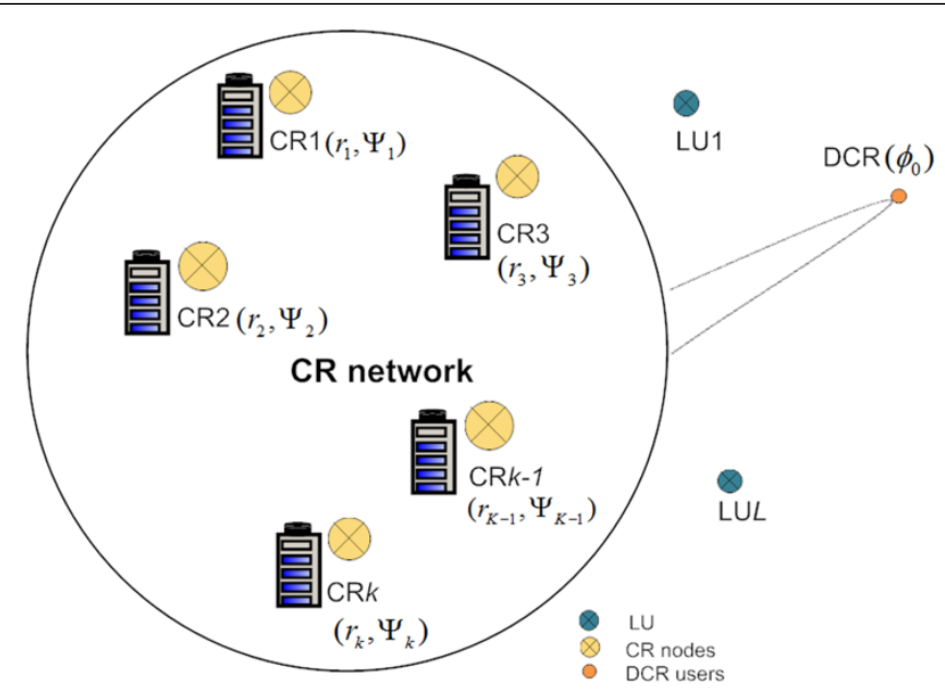

Figure 6 Power consumption of CR network with PODB method.

Thus, it has been proved that the life time of the network adopting PODB method is longer than that adopting null-steering method. Only when $K_{0}=1$, the network has the same lifetime by employing both two DB methods.

\section{Conclusions}

A new DB method, which is called PODB method, has been proposed in this article in the application of a green $\mathrm{CR}$ network. It is implemented in a way that $\mathrm{CR}$ nodes transmit signals constructively towards directions of DCR users and destructively to the LU. Considering the average beam pattern of the PODB method, it achieves the highest gain at the direction of DCR users and the lowest to the LU. The PODB method can be computed at each node in a so called distributed way, i.e., each node is only aware of its own location, and the directions of LU and DCR users. The PODB is different from the null-steering-based DB method meaning that the weights of the former can be regarded as a phase perturbation of each node. Consequently, it utilizes energy of the whole network evenly and effectively, a characteristic which renders itself to green concept.

In a CR network, it is extremely important to calculate the probability of the case that the power transmitted by the CR network towards directions of $\mathrm{LU}$ is lower than a certain threshold. Therefore, the CDF of the beam pattern of the PODB method has been derived in this article assuming a large number of $C R$ nodes. Comparing with the DB method without particular nulling directions, the PODB method achieves much higher chances of transmitting less power towards directions of LU. To increase the chances that the disturbances to LU, which are caused by the CR network are less than the interference power threshold that the LU can tolerate, the presented CDF suggests that more $C R$ nodes should participate in the $C R$ network to perform DB.

\section{Appendix 1}

Proof of Theorem 1

It has been discussed in [23] that

$$
\frac{1}{K} \mathbf{u}_{m}^{H} \mathbf{u}_{n}=\frac{1}{K} \sum_{k=1}^{K} e^{-j \alpha_{m, n} z_{k}} \stackrel{P 1}{\rightarrow} E\left[e^{-j \alpha_{m, n} z_{k}}\right]=\left\{\begin{array}{c}
1, m=n \\
\frac{2 J_{1}\left(\alpha_{m, n}\right)}{\alpha_{m, n}}, m \neq n
\end{array}\right.
$$

where $z_{k} \triangleq \frac{r_{k}}{R} \sin \frac{\phi_{m}+\phi_{n}-2 \Psi_{k}}{2}$, and the pdf of $z_{k}$ has been presented in [6],

$$
\begin{aligned}
& f_{z}\left(z_{k}\right)=\frac{2}{\pi} \sqrt{1-z_{k^{\prime}}^{2}},\left|z_{k}\right| \leq 1 \\
& \frac{1}{K} \mathbf{u}_{m}^{H} \mathbf{u}_{n}^{*}=\frac{1}{K} \sum_{k=1}^{K} e^{-j \frac{2 \pi}{\lambda} r_{k}\left[\cos \left(\phi_{n}-\Psi_{k}\right)+\cos \left(\phi_{m}-\Psi_{k}\right)-2 \cos \left(\phi_{0}-\Psi_{k}\right)\right]}
\end{aligned}
$$

The above exponential item can be further simplified by the following

$$
\begin{aligned}
& \cos \left(\phi_{n}-\Psi_{k}\right)+\cos \left(\phi_{m}-\Psi_{k}\right)-2 \cos \left(\phi_{0}-\Psi_{k}\right) \\
& =2 \cos \frac{\phi_{n}+\phi_{m}-2 \Psi_{k}}{2} \cos \frac{\phi_{n}-\phi_{m}}{2}-2 \cos \left(\frac{\phi_{n}+\phi_{m}-2 \Psi_{k}}{2}+\phi_{0}-\frac{\phi_{n}+\phi_{m}}{2}\right) \\
& =2 a_{1} \cos \frac{\phi_{n}+\phi_{m}-2 \Psi_{k}}{2}+2 a_{2} \sin \frac{\phi_{n}+\phi_{m}-2 \Psi_{k}}{2}
\end{aligned}
$$

where

$$
\begin{aligned}
& a_{1}=\cos \frac{\phi_{n}-\phi_{m}}{2}-\cos \left(\phi_{0}-\frac{\phi_{n}+\phi_{m}}{2}\right) \\
& a_{2}=\sin \left(\phi_{0}-\frac{\phi_{n}+\phi_{m}}{2}\right)
\end{aligned}
$$




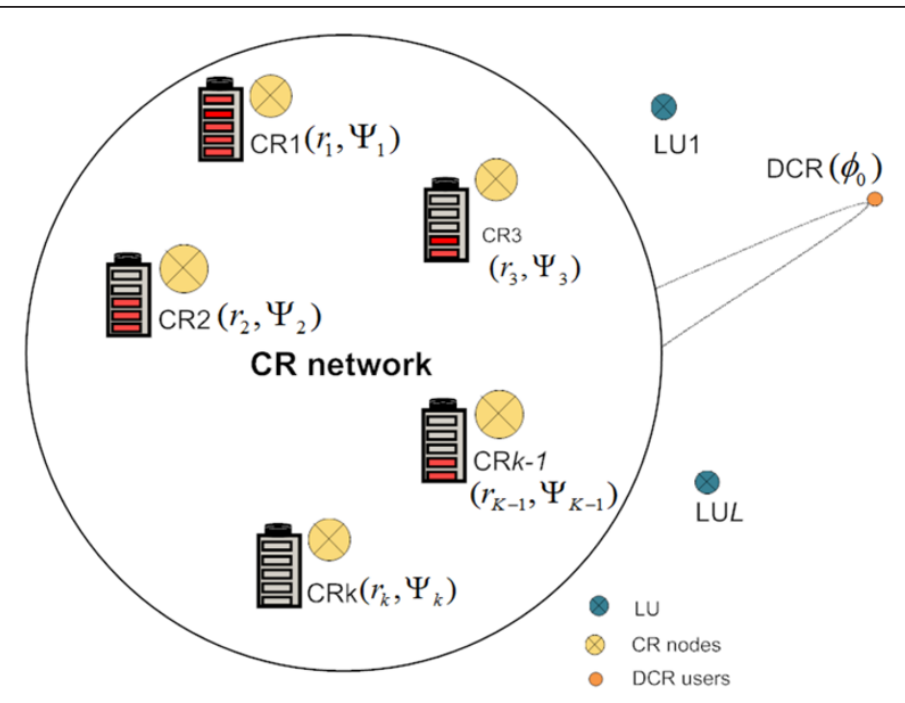

Figure 7 Power consumption of CR network with null-steering DB method.

We define

$$
\begin{aligned}
& a \triangleq \sqrt{a_{1}^{2}+a_{2}^{2}}=\sqrt{1+\cos ^{2} \frac{\phi_{m}-\phi_{n}}{2}-\cos \left(\phi_{0}-\phi_{n}\right)-\cos \left(\phi_{0}-\phi_{m}\right)} \\
& \theta \triangleq \tan ^{-1} \frac{a_{1}}{a_{2}}
\end{aligned}
$$

Equation (43) can be written as

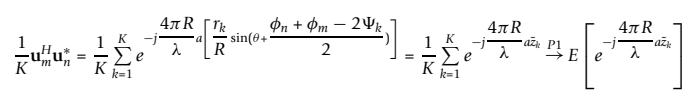

where

$$
\tilde{z}_{k}=\frac{r_{k}}{R} \sin \left(\theta+\frac{\phi_{n}+\phi_{m}-2 \Psi_{k}}{2}\right)=\frac{r_{k}}{R} \sin \left(\theta+\frac{\phi_{n}+\phi_{m}}{2}-\Psi_{k}\right)
$$

$\tilde{z}_{k}$ has the same distribution with $z_{k}$, because the pdf of $z_{k}$ in (42) has nothing to do with $\varphi_{m}, \varphi_{n}$. Therefore, using the same method, (45) can be further written as

$$
\frac{1}{K} \mathbf{u}_{m}^{H} \mathbf{u}_{n}^{*} \stackrel{P 1}{\rightarrow} E\left[e^{-j \frac{4 \pi R}{\lambda} a \tilde{z}_{k}}\right]=\frac{2 J_{1}\left(\tilde{\alpha}_{m, n}\right)}{\tilde{\alpha}_{m, n}}
$$

where

$$
\tilde{\alpha}_{m, n}=4 \pi \frac{R}{\lambda} a=4 \pi \frac{R}{\lambda} \sqrt{1+\cos ^{2} \frac{\phi_{m}-\phi_{n}}{2}-\cos \left(\phi_{0}-\phi_{n}\right)-\cos \left(\phi_{0}-\phi_{m}\right)}
$$

Especially, when $m=n$,

$$
E\left[\left.e^{-j \frac{4 \pi R}{\lambda} a \tilde{z}_{k}}\right|_{m=n}\right]=E\left[e^{-j 2 \alpha_{m, 0} z_{k}}\right]=\frac{J_{1}\left(2 \alpha_{m, 0}\right)}{\alpha_{m, 0}}
$$

Equations (41), (47) and (49) show the results of Theorem 1.

\section{Appendix 2}

Proof of Theorem 2

Based on the definition of $\boldsymbol{\Gamma}$,

$$
\boldsymbol{\Gamma}=\left(\begin{array}{l}
\mathbf{X}^{H} \mathbf{X} \mathbf{X}^{H} \mathbf{Y} \\
\mathbf{Y}^{H} \mathbf{X} \mathbf{Y}^{H} \mathbf{Y}
\end{array}\right)
$$

and

$$
\boldsymbol{\Gamma}_{1}=\frac{1}{K} E\left[\mathbf{X}^{H} \mathbf{X}\right]
$$

$$
\boldsymbol{\Gamma}_{2}=\frac{1}{K} E\left[\mathbf{Y}^{H} \mathbf{Y}\right]
$$

Using the results in Theorem 1,

$$
\begin{aligned}
& \left(\boldsymbol{\Gamma}_{1}\right)_{m, n}=E\left[\frac{\left.\mathbf{x}_{m}^{H} \mathbf{x}_{n}\right]}{K}\right]=E\left[\frac{\left(\mathbf{u}_{m}^{H}+\mathbf{u}_{m}^{T}\right)\left(\mathbf{u}_{n}+\mathbf{u}_{n}^{*}\right)}{4 K}\right] \\
& =\left\{\begin{array}{c}
\frac{J_{1}\left(2 \alpha_{m, 0}\right)}{2 \alpha_{m, 0}}+\frac{1}{2}, m=n=1,2, \ldots, M \\
\frac{J_{1}\left(\tilde{\alpha}_{m, n}\right)}{\tilde{\alpha}_{m, n}}+\frac{J_{1}\left(\alpha_{m, n}\right)}{\alpha_{m, n}}, m \neq n ; m, n=1,2, \ldots, M
\end{array}\right.
\end{aligned}
$$

$$
\frac{1}{K} E\left[\left(\mathbf{X}^{H} \mathbf{Y}\right)_{m, n}\right]=E\left[\frac{\mathbf{x}_{m}^{H} \mathbf{y}_{n}}{K}\right]=\frac{1}{K} E\left[\frac{\left(\mathbf{u}_{m}^{H}+\mathbf{u}_{m}^{T}\right)\left(\mathbf{u}_{n}-\mathbf{u}_{n}^{*}\right)}{j 4 K}\right]=0
$$

$$
\frac{1}{K} E\left[\left(\mathbf{Y}^{H} \mathbf{X}\right)_{m, n}\right]=E\left[\left(\frac{\mathbf{x}_{m}^{H} \mathbf{y}_{n}}{K}\right)^{H}\right]=0
$$




$$
\begin{aligned}
& \left(\boldsymbol{\Gamma}_{2}\right)_{m, n}=E\left[\frac{\mathbf{y}_{m}^{H} \mathbf{y}_{n}}{K}\right]=E\left[\frac{\left(\mathbf{u}_{m}^{H}+\mathbf{u}_{m}^{T}\right)\left(\mathbf{u}_{n}+\mathbf{u}_{n}^{*}\right)}{4 K}\right] \\
& =\left\{\begin{array}{c}
\frac{1}{2}-\frac{J_{1}\left(2 \alpha_{m, 0}\right)}{2 \alpha_{m, 0}}, m=n=1,2, \ldots, M \\
\frac{J_{1}\left(\alpha_{m, n}\right)}{\alpha_{m, n}}-\frac{J_{1}\left(\tilde{\alpha}_{m, n}\right)}{\tilde{\alpha}_{m, n}}, m \neq n ; m, n=1,2, \ldots, M
\end{array}\right.
\end{aligned}
$$

Equations (53), (54), (55) and (56) demonstrate the results of Theorem 2 .

\section{Appendix 3}

Proof of Theorem 3

Based on Equations (7) and (10)

$$
\tilde{P}\left(\phi_{\bullet}\right)=\frac{1}{K^{2}} E\left[\left|\sum_{k=1}^{K} e^{-j \alpha_{0}, z_{k}+j \mu_{k}}\right|^{2}\right]=\frac{1}{K^{2}}\left(K+E\left[\sum_{k=1}^{K} \sum_{l=1, l \neq k}^{K} e^{-j \alpha_{\bullet}\left(z_{k}-z_{l}\right)+j\left(\mu_{k}-\mu_{l}\right)}\right]\right)
$$

As mentioned before, $\mu_{k}$ is a small perturbation of the phase of each node. We here adopt the approximation of $e^{x}$ by $e^{x} \approx 1+x$, and it holds the following result [31],

$$
E\left[\left|e^{j x}-(1+j x)\right|\right] \leq E\left[\frac{|j x|^{2}}{2}\right]=\frac{E\left[|x|^{2}\right]}{2}
$$

Then we have

$$
e^{j\left(\mu_{k}-\mu_{l}\right)} \approx 1+j\left(\mu_{k}-\mu_{l}\right)
$$

Equation (57) can be approximated by

$$
\tilde{P}\left(\phi_{\bullet}\right) \approx \tilde{P}_{a}\left(\phi_{\bullet}\right)=\frac{1}{K^{2}}\left(K+\sum_{k=1}^{K} \sum_{l=1, l \neq k}^{K} E\left[e^{-j \alpha_{\bullet}, 0 z_{k}} e^{j \alpha_{\bullet}, 0 z_{l}}\left(1+j \mu_{k}-j \mu_{l}\right)\right]\right)
$$

We define a new random variable

$$
\xi_{k} \triangleq e^{-j \alpha_{\bullet}, 0 z_{k}} \mu_{k}
$$

Based on the pdf of $z_{k}$, we have the following results [6]

$$
E\left[\left(u_{\bullet}\right)_{k}\right]=E\left[e^{-j \alpha_{\bullet}, 0 z_{k}}\right]=E\left[\left(u_{\bullet}^{*}\right)_{k}\right]=\left\{\begin{array}{c}
1, \phi_{\bullet}=\phi_{0} \\
\frac{2 J_{1}\left(\alpha_{\bullet, 0}\right)}{\alpha_{\bullet}, 0}, \phi_{\bullet}=\phi_{0}
\end{array}\right.
$$

Using the result in Theorem 1, it is easy to calculate the expectations of the following variables,

$$
\begin{gathered}
E\left[\left(\boldsymbol{u}_{\bullet}\right)_{k}\left(\boldsymbol{u}_{m}^{*}\right)_{k}\right]=\left\{\begin{array}{c}
1, \phi_{\bullet}=\phi_{m}, m=1,2, \ldots, M \\
\frac{2 J_{1}\left(\alpha_{\bullet, m}\right)}{\alpha_{\bullet, m}}, \phi_{\bullet}=\phi_{m}, m=1,2, \ldots, M
\end{array}\right. \\
E\left[\left(\boldsymbol{u}_{\bullet}\right)_{k}\left(\boldsymbol{u}_{m}\right)_{k}\right]=\left\{\begin{array}{l}
\frac{J_{1}\left(2 \alpha_{m, 0}\right)}{\alpha_{m, 0}}, \phi_{\bullet}=\phi_{m}, m=1,2, \ldots, M \\
\frac{2 J_{1}(\tilde{\alpha} \bullet, m)}{\tilde{\alpha} \bullet, m}, \phi_{\bullet}=\phi_{m}, m=1,2, \ldots, M
\end{array}\right.
\end{gathered}
$$

Consequently from Equations (63), (64) and (23), the expectation of $\xi_{k}$ can be calculated by

$$
E\left[\xi_{k}\right]=E\left[e^{-j \alpha_{\bullet}, 0 z_{k}} \mu_{k}\right]=\sum_{m=1}^{M} q_{m} E\left[\frac{\left(\boldsymbol{u}_{\bullet}\right)_{k}\left(\boldsymbol{u}_{m}\right)_{k}-\left(\boldsymbol{u}_{\bullet}\right)_{k}\left(\boldsymbol{u}_{m}^{*}\right)_{k}}{2 j}\right]=\sum_{m=1}^{M} \frac{q_{m}}{2 j} \zeta_{\bullet}, m
$$

where

$$
\zeta_{\bullet, m}=\left\{\begin{array}{c}
\frac{J_{1}\left(2 \alpha_{m, 0}\right)}{\alpha_{m, 0}}-1, \phi_{\bullet}=\phi_{m}, m=1,2, \ldots, M \\
\frac{2 J_{1}\left(\tilde{\alpha}_{\bullet, m}\right)}{\tilde{\alpha}_{\bullet}, m}-\frac{2 J_{1}\left(\alpha_{\bullet}, m\right)}{\alpha_{\bullet}, m}, \phi_{\bullet} \neq \phi_{m}, m=1,2, \ldots, M
\end{array}\right.
$$

From Equation (65), it is easy to verify that

$$
E\left[\xi_{k}^{*}\right]=-E\left[\xi_{k}\right]
$$

Meanwhile, $e^{j \alpha_{\bullet}, 0 z_{k}}$ and $e^{j \alpha_{\bullet}, 0} z_{l}, l \neq k$ are identical independently distributed variables. $\xi_{k}$ and $e^{j \alpha_{\bullet}, 0} z_{l}, l \neq k$ are independently distributed random variables. Thus, with the result in Equations (62) and (65), considering the general case that $\varphi_{.} \neq \varphi_{0}$, we can deduce the second item in Equation (60) further by

$$
\begin{aligned}
& \sum_{k=1}^{K} \sum_{l=1, l \neq k}^{K} E\left[e^{-j \alpha_{\omega_{0}, 0 z_{k}}} e^{j \alpha_{\bullet}, 0 z_{l}}\left(1+j \mu_{k}-j \mu_{l}\right)\right] \\
& =\left(K^{2}-K\right)\left[\left(\frac{2 J_{1}\left(\alpha_{\bullet}, 0\right)}{\left.\alpha_{\bullet}\right)}\right)^{2}+j E\left[\xi_{k}\right] \frac{2 J_{1}\left(\alpha_{\bullet, 0}\right)}{\alpha_{\bullet} 0}-j E\left[\xi_{l}^{*}\right] \frac{2 J_{1}\left(\alpha_{\bullet, 0}\right)}{\alpha_{\bullet}, 0}\right] \\
& =\left(K^{2}-K\right)\left[\left(\frac{2 J_{1}\left(\alpha_{\bullet}, 0\right)}{\alpha_{\bullet}, 0}\right)^{2}+\frac{2 J_{1}\left(\alpha_{\bullet}, 0\right)}{\alpha_{\bullet}, 0} \sum_{m=1}^{M} q_{m} \zeta_{\bullet}, m\right]
\end{aligned}
$$

Substituting Equation (68) into Equation (60), we have final results

$$
\tilde{P}\left(\phi_{\bullet}\right) \approx \tilde{P}_{a}\left(\phi_{\bullet}\right)=\frac{1}{K}+\frac{K-1}{K}\left[\left(\frac{2 J_{1}\left(\alpha_{\bullet}\right)}{\left.\alpha_{\bullet}\right)}\right)^{2}+\frac{J_{1}\left(\alpha_{\bullet}, 0\right)}{\left.\alpha_{\bullet}\right)} \sum_{m=1}^{M} q_{m} \zeta_{\bullet}, m\right]
$$

The error of the approximation in (61) can be bounded using the result of (58) by

$$
\begin{aligned}
& E\left[\left|e^{j\left(\mu_{k}-\mu_{l}\right)}-1-j \mu_{k}+j \mu_{l}\right|\right] \leq \frac{1}{2} E\left[\left|\mu_{k}-\mu_{l}\right|^{2}\right]=E\left[\mu_{k}^{2}\right]-\left(E\left[\mu_{k}\right]\right)^{2} \\
& E\left[\left(\mathbf{y}_{\bullet}\right)_{k}\right]=E\left[\frac{\left(\mathbf{u}_{\bullet}\right)_{k}-\left(\mathbf{u}_{\bullet}\right)_{k}^{*}}{2 j}\right]=0
\end{aligned}
$$

Based on Equation (23) and the results of Equation (62), we can deduce that

$$
E\left[\mu_{k}\right]=\mathbf{c} \boldsymbol{\Gamma}_{2}^{-1} E\left[\left(\mathbf{y}_{1}\right)_{k},\left(\mathbf{y}_{2}\right)_{k}, \ldots,\left(\mathbf{y}_{M}\right)_{k}\right]^{H}=0
$$

In Theorem 2, it holds that

$$
E\left[\left(\mathbf{y}_{m}^{*}\right)_{k}\left(\mathbf{y}_{n}\right)_{k}\right]=\left(\boldsymbol{\Gamma}_{2}\right)_{m, n}
$$

Therefore,

$$
E\left\{\left[\left(\mathbf{y}_{1}\right)_{k},\left(\mathbf{y}_{2}\right)_{k}, \ldots,\left(\mathbf{y}_{M}\right)_{k}\right]^{H}\left[\left(\mathbf{y}_{1}\right)_{k},\left(\mathbf{y}_{2}\right)_{k}, \ldots,\left(\mathbf{y}_{M}\right)_{k}\right]\right\}=\boldsymbol{\Gamma}_{2}(74)
$$




$$
E\left[\mu_{k}^{2}\right]=E\left[\left(\mathbf{c} \Gamma_{2}^{-1}\left[\left(\mathbf{y}_{1}\right)_{k^{\prime}}\left(\mathbf{y}_{2}\right)_{k^{\prime}}, \ldots,\left(\mathbf{y}_{M}\right)_{k}\right]^{H}\right)^{2}\right]=\mathbf{c} \boldsymbol{\Gamma}_{2}^{-1} \mathbf{c}^{H}(75)
$$

By substituting Equations (75), (72) into (70), we can write

$$
E\left[e^{j\left(\mu_{k}-\mu_{l}\right)}-1-j \mu_{k}+j \mu_{l}\right] \leq \mathbf{c} \Gamma_{2}^{-1} \mathbf{c}^{H}
$$

The approximation error in the second item of Equation (60) can be written

$$
E\left[e^{-j \mu_{\alpha}\left(z_{k}-z\right)}\left(e^{j\left(\mu_{k}-\mu_{i}\right)}-1-j \mu_{k}+j \mu_{1}\right) \mid\right]=E\left[e^{j\left(\mu_{k}-\mu_{i}\right)}-1-j \mu_{k}+j \mu_{k} \mid\right] \leq \mathbf{c} \Gamma_{2}^{-1} \mathbf{c}^{H}
$$

Then the estimation error $\varepsilon$ between $\tilde{P}_{a}\left(\phi_{\bullet}\right)$ and $\tilde{P}\left(\phi_{\bullet}\right)$ can be written based on the result of Equation (77)

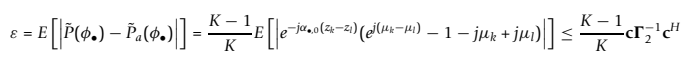

\section{Appendix 4}

Proof of Equation (27)

When $\varphi=\varphi_{0}$, the average beam pattern at $\varphi_{0}$ can be written as:

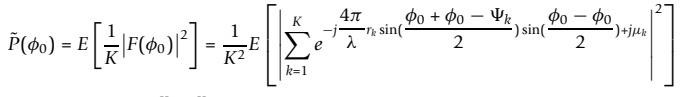

$$
\begin{aligned}
& =\frac{1}{K}+\frac{1}{K^{2}} \sum_{k=1}^{K} \sum_{l=1,1, f k}^{K} E\left[e^{i\left(\mu_{k}-\mu_{l}\right)}\right]
\end{aligned}
$$

Using the same approximation method in Equation (59)

$$
\tilde{P}\left(\phi_{0}\right) \approx \frac{1}{K}+\frac{1}{K^{2}} \sum_{k=1}^{K} \sum_{l=1, l \neq k}^{K} E\left[1+j \mu_{k}-j \mu_{l}\right]
$$

Based on the result in Equation (72), we can further write

$$
E\left[1+j \mu_{k}-j \mu_{l}\right]=1
$$

Substituting Equation (81) into (80), we have the result of Equation (27)

$$
\tilde{P}\left(\phi_{0}\right) \approx \frac{1}{K}+\frac{1}{K^{2}}\left(K^{2}-K\right)=1
$$

\section{Appendix 5}

Proof of Equation (28)

Theorem 3 and the definition of $\boldsymbol{\Gamma}_{2}$ in Theorem 2 illustrate that, when $\varphi_{\bullet}=\varphi_{m}, m=1,2, \ldots, M$,

$$
\zeta_{\bullet},\left.n\right|_{\phi_{\bullet}=\phi_{m}}=2\left(\boldsymbol{\Gamma}_{2}\right)_{m, n}, m, n=1,2, \ldots, M
$$

Therefore,

$$
\left(\varsigma_{m, 1}, \zeta_{m, 2}, \ldots, \zeta_{m, M}\right)^{T}=-2\left(\boldsymbol{\Gamma}_{2}\right)^{T} \mathbf{v}_{m}=-2 \boldsymbol{\Gamma}_{2} \mathbf{v}_{m}, m=1,2, \ldots, M
$$

where $\mathbf{v}_{m}$ denotes a $M \times 1$ vector with ones as the entities of the $m$ th row.

Meanwhile based on the definition of $\left(q_{1}, q_{2}, \ldots, q_{M}\right)$ we can write when $\varphi_{\bullet}=\varphi_{m}, m=1,2, \ldots, M$,

$$
\sum_{n=1}^{M} \frac{q_{n}}{2} \zeta_{\bullet},\left.n\right|_{\phi_{\bullet}=\phi_{m}}=-\mathbf{c} \boldsymbol{\Gamma}_{2}^{-1} \boldsymbol{\Gamma}_{2} \mathbf{v}_{m}=-c_{m}=-\frac{2 J_{1}\left(\alpha_{m, 0}\right)}{\alpha_{m, 0}}
$$

By substituting (85) into the average beam pattern in Theorem 3,

$$
\tilde{P}\left(\phi_{m}\right) \approx \frac{1}{K}+\frac{K-1}{K}\left[\left(\frac{2 J_{1}\left(\alpha_{m, 0}\right)}{\alpha_{m, 0}}\right)^{2}-\frac{2 J_{1}\left(\alpha_{m, 0}\right)}{\alpha_{m, 0}} \frac{2 J_{1}\left(\alpha_{m, 0}\right)}{\alpha_{m, 0}}\right]=\frac{1}{K}
$$

Which shows the result of Equation (28).

\section{Appendix 6}

Proof of Theorem 4

$$
P\left(\phi_{\bullet}\right)=\frac{1}{K^{2}}\left|\sum_{k=1}^{K} e^{-j \alpha_{\bullet}, 0 z_{k}+j \mu_{k}}\right|^{2}
$$

We define a new random variable

$$
\chi \triangleq \sum_{k=1}^{K} \frac{1}{K} e^{-j \omega_{0}, z_{k}+j \mu_{k}} \approx \sum_{k=1}^{K} \frac{\left(1+j \mu_{k}\right) e^{-j \omega_{0}, z_{k}}}{K}=\sum_{K=1}^{K} \frac{e^{-j \omega_{0}, z_{k}}+j \xi_{k}}{K}
$$

Definition of $\xi_{k}$ is shown in Appendix 3 Equation (61). $e^{-j \alpha_{\boldsymbol{0}, 0} z_{k}}+j \xi_{k}$ and $e^{-j \alpha_{0,0} z_{l}}+j \xi_{l}, l \neq k$ are identical independently distributed variables. Thus, if $K$ is large enough, according to the Central Limit Theorem, $\chi$ has a Gaussian distribution, i.e. $\chi \sim N\left(\rho, \sigma^{2}\right)$, where

$$
\begin{aligned}
& \rho=E[\chi]=E\left[e^{-j \alpha_{\boldsymbol{\theta}, 0} z_{k}}+j \xi_{k}\right] \\
& \sigma^{2}=D[\chi]=\frac{1}{K} D\left[e^{-j \boldsymbol{\omega}_{0}, 0 z_{k}}+j \xi_{k}\right]
\end{aligned}
$$

Using the result discussed in Equations (62) and (65) in Appendix 3

$$
\rho=E\left[e^{-j \alpha_{\bullet}, 0 z_{k}}+j \xi_{k}\right]=\frac{2 J\left(\alpha_{\bullet}\right)}{\alpha_{\bullet}, 0}+\sum_{m=1}^{M} \frac{q_{m}}{2} \zeta_{\bullet}, m
$$

From Equation (75) in Appendix 3,

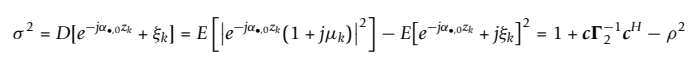

Thus, by adopting the results shown in (91) and (92),

$$
\chi \sim N\left(\rho, \frac{1+c \boldsymbol{\Gamma}_{2}^{-1} \boldsymbol{c}^{H}-\rho^{2}}{K}\right)
$$


From Equation (93), if we define a new random variable $\gamma$ by $\gamma \triangleq|\chi-\rho|$, the distribution of $\gamma$ is Rayleigh distribution, i.e. $\gamma \sim \operatorname{Rayleigh}\left(\sigma_{0}^{2}\right)$, where $\sigma_{0}^{2}=\frac{\sigma^{2}}{2}$. We then are able to calculate the probability $\operatorname{Prob}\left\{P\left(\varphi_{.}\right) \mid P\right.$ $\left.(\varphi.) \leq P_{0}\right\}$. We next discuss the outcome for the different cases.

$$
\begin{aligned}
& \text { If }|\rho|>\sqrt{P_{0}}, \gamma \in\left[|\rho|-\sqrt{P_{0}},|\rho|+\sqrt{P_{0}}\right] \\
& \text { then } \\
& \operatorname{Prob}\left\{P\left(\phi_{\bullet}\right) \mid P\left(\phi_{\bullet}\right) \leq P_{0}\right\}=F\left(|\rho|+\sqrt{P_{0}}\right)-F\left(|\rho|+\sqrt{P_{0}}\right) \\
& \text { If }|\rho|<\sqrt{P_{0}}, \gamma \in\left[0,|\rho|+\sqrt{P_{0}}\right] \\
& \text { then }
\end{aligned}
$$

$$
\operatorname{Prob}\left\{P\left(\phi_{\bullet}\right) \mid P\left(\phi_{\bullet}\right) \leq P_{0}\right\}=F\left(|\rho|+\sqrt{P_{0}}\right)
$$

where

$$
F\left(\gamma_{0}\right)=\operatorname{Prob}\left(\gamma \mid \gamma \leq \gamma_{0}\right)=1-\exp \left(-\frac{\gamma_{0}^{2}}{2 \sigma_{0}^{2}}\right)=1-\exp \left[-\gamma_{0}^{2} K /\left(1+\mathbf{c}_{2}^{-1} \mathbf{c}^{H}-\rho^{2}\right)\right]
$$

By combining (94) and (95), it is easy to verify Theorem 4.

$$
\operatorname{Prob}\left\{P\left(\phi_{\bullet}\right) \mid P\left(\phi_{\bullet}\right) \leq P_{0}\right\}=F\left(|\rho|+\sqrt{P_{0}}\right)-\frac{\operatorname{sgn}\left(|\rho|+\sqrt{P_{0}}\right)+1}{2} F\left(|\rho|+\sqrt{P_{0}}\right) .
$$

\section{Appendix 7}

\section{Proof of Equation (31)}

Using the result of Equation (59) in appendix E, we can write

$$
\left.\rho\right|_{\phi_{\bullet}=\phi_{m}}=\frac{2 J\left(\alpha_{m, 0}\right)}{\alpha_{m, 0}}+\sum_{n=1}^{M} \frac{q_{n}}{2} \varsigma_{m, n}=0
$$

As a result,

$$
\operatorname{Prob}\left\{P\left(\phi_{m}\right) \mid P\left(\phi_{m}\right) \leq P_{0}\right\}=F\left(\sqrt{P_{0}}\right)=1-\exp \left[-P_{0} K /\left(1+\mathbf{c r}_{2}^{-1} \mathbf{c}^{H}\right)\right]
$$

\section{Competing interests}

The authors declare that they have no competing interests.

Received: 1 October 2011 Accepted: 27 February 2012 Published: 27 February 2012

\section{References}

1. J Mitola, Cognitive radio: making software radios more personal. IEEE Person Commun. 6(4), 13-18 (1999). doi:10.1109/98.788210

2. J Mitola, Dissertation, Royal Inst. Technol. (KTH) (2000)

3. S Haykin, Cognitive radio: brain-empowered wireless communications. IEEE J Sel Area Commun. 23(2), 201-210 (2005)

4. X Lian, H Nikookar, J Zhou, Adaptive robust beamformers for cognitive radio, in Proc IEEE Wireless Technology (EuWiT), Amsterdam, The Netherlands, pp. 103-106

5. X Lian, H Nikookar, LP Ligthart, Adaptive OFDM beamformer with constrained weights for cognitive radio, in Proc IEEE 69th Vehicular
Technology Conference (VTC Spring), Bacelona, Spain, pp. 1-5 (2009). doi:10.1109/NETECS.2009.5073362

6. H Ochiai, P Mitran, HV Poor, V Tarokh, Collaborative beamforming for distributed wireless ad hoc sensor networks. IEEE Trans Signal Process. 53(11), 4110-4124 (2005)

7. L Dong, AP Petropulu, HV Poor, A cross-layer approach to collaborative beamforming for wireless ad hoc networks. IEEE Trans Signal Process. 56(7), 2981-2993 (2008)

8. K Zarifi, S Affes, A Ghrayeb, Distributed beamforming for wireless sensor networks with random node location, in Proc Int Conf Acoustics, Speech, and Signal Process (ICASSP'09), Taipei, Taiwan, pp. 2261-2264 (2009). doi:10.1109/ ICASSP. 2009.4960070

9. R Mudumbai, G Barriac, U Madhow, On the feasibility of distributed beamforming in wireless sensor networks. IEEE Trans Wirel Commun. 6(5), 1754-1763 (2007)

10. Y Jing, H Jafarkhani, Network beamforming using relays with perfect channel information, in Proc Int Conf Acoustics, Speech, and Signal Process (ICASSP'07), Hawaii, USA, pp. 473-476 (2007). doi:10.1109/ ICASSP.2007.366575

11. G Zheng, KK Wong, A Paulraj, B Ottersten, Collaborativerelay beamforming with perfect CSI: optimum and distributed implementations. IEEE Signal Process Lett. 16(4), 257-260 (2009)

12. V Havary-Nassab, S Shahbazpanahi, A Grami, ZQ Luo, Distributed beamforming for relay networks based on second-order statistics of the channel state information. IEEE Trans Signal Process. 56(9), 4306-4316 (2008)

13. S Fazeli-Dehkordy, S Shahbazpanahi, S Gazor, Multiple peer-to-peer communications using a network of relays. IEEE Trans Signal Process. 57(8), 3053-3062 (2009)

14. H Chen, AB Gershman, S Shahbazpanahi, Filter-and-forward distributed beamforming in relay networks with frequency selective fading. IEEE Trans Signal Process. 58(3), 1251-1262 (2010)

15. DR Brown III, HV Poor, Time-slotted round-trip carrier synchronization for distributed beamforming. IEEE Trans Signal Process. 56(11), 5630-5643 (2008)

16. JA Bucklew, WA Sethares, Convergence of a class of decentralized beamforming algorithms. IEEE Trans Signal Process. 56(6), 2280-2288 (2008)

17. R Mudumbai, DR Brown, U Madhow, HV Poor, Distributed transmit beamforming: challenges and recent progress. IEEE Commun Mag. 47(2), 102-110 (2009)

18. GL Stuber, Principles of Mobile Communication (Kluwer, Boston, MA, 1996)

19. IS Sadowsky, V Kafedziski, On the correlation and scattering functions of the WSSUS channel for mobile communication. IEEE Trans Veh Technol. 47(1), 270-282 (2008)

20. A Abdi, JA Barger, M Kaveh, A parametric model for the distribution of the angle of arrival and the associated correlation function and power spectrum at the mobile station. IEEE Trans Veh Technol. 51(3), 425-434 (2002). doi:10.1109/TVT.2002.1002493

21. Y Deng, AG Buur, D Pearce, D Grace, Distributed beamforming for cognitive radio networks, in Proc Communications and Networking in China (CHINACOM'08), Hangzhou, China, pp. 1206-1210 (2008). doi:10.1109/ CHINACOM.2008.4685243

22. J Liu, W Chen, Z Cao, Y Zhang, A distributed beamforming approach for enhanced opportunistic spectrum access in cognitive radios, in Proc Global Telecommunications Conference (Globecom), Hawaii, USA, pp. 1-6 (2009). doi:10.1109/GLOCOM.2009.5425809

23. K Zarif, S Affes, A Ghrayeb, Collaborative null-steering beamforming for uniformly distributed wireless sensors networks. IEEE Trans Signal Process. 58(3), 1889-1903 (2010)

24. I Budiarjo, MK Lakshmanan, H Nikookar, Cognitive radio dynamic access technique. Wirel Person Commun. 45(3), 293-324 (2008). doi:10.1007/ s11277-008-9473-6

25. X Lian, H Nikookar, LP Ligthart, Efficient radio transmission with adaptive and distributed beamforming for intelligent WiMAX. J Wirel Person Commun. 53(3), 405-431 (2011)

26. H Steyskal, Simple method for pattern nulling by phase perturbation. IEEE Trans Antenna Propagat. 31(1), 163-166 (1983). doi:10.1109/ TAP 1983.1142994

27. RJ Mailloux, Covariance matrix augmentation to produce adaptive array pattern troughs. Electron Lett. 31(10), 771-772 (1998) 
28. M Zatman, Production of adaptive array troughs by dispersion synthesis Electron Lett. 31(25), 2141-2142 (1998)

29. JR Guerci, Theory and application of covariance matrix tapers for robust adaptive beamforming. IEEE Trans Signal Process. 47(4), 977-985 (1999). doi:10.1109/78.752596

30. J Riba, J Goldberg, G Vazquez, Robust beamforming for interference rejection in mobile communications. IEEE Trans Signal Process. 45(1), 271-275 (1997). doi:10.1109/78.552229

31. P Billingsley, Probability and Measure (John Wiley \& Sons, New York, NY, 1995)

doi:10.1186/1687-1499-2012-65

Cite this article as: Lian et al: Distributed beam forming with phaseonly control for green cognitive radio networks. EURASIP Journal on Wireless Communications and Networking 2012 2012:65.

Submit your manuscript to a SpringerOpen ${ }^{\mathcal{O}}$ journal and benefit from:

- Convenient online submission

- Rigorous peer review

- Immediate publication on acceptance

- Open access: articles freely available online

- High visibility within the field

- Retaining the copyright to your article

Submit your next manuscript at $>$ springeropen.com 